\title{
Synthesis and properties on polyaniline in the presence of nickel chloride
}

\author{
Y. Tan, Y. Zhang, J. Kan* \\ School of Chemistry and Chemical Engineering, Yangzhou University, Yangzhou, 225002, PR China
}

Received 18 January 2009; accepted in revised form 25 March 2009

\begin{abstract}
Both Conducting and Ferromagnetic polyaniline containing nickel (abbreviated as PANI-Ni) is synthesized in a solution containing aniline, $\mathrm{HCl}$ and $\mathrm{NiCl}_{2}$, using peroxydisulphate as an oxidant and horseradish peroxidase as a catalyst. The conductivity of PANI-Ni is about $10^{-1} \mathrm{~S} \mathrm{~cm}^{-1}$. The result of electron paramagnetic resonance (EPR) spectra indicated

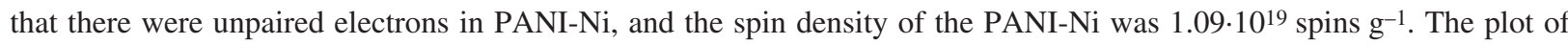
magnetization $(M)$ against the magnetic field $(H)$ indicated that the PANI-Ni was soft ferromagnetic at about $300 \mathrm{~K}$, the saturation magnetization $\left(M_{s}\right)$ and the coercive force $\left(H_{c}\right)$ of which were $4.5 \mathrm{emu} \mathrm{g}^{-1}$ (electromagnetic unit per gram) and about 55 Oe, respectively. UV-Visible (UV-vis) and Fourier Transform Infrared (FTIR) spectra showed that there was strong interaction between $\mathrm{Ni}^{2+}$ and PANI chains, but the backbone chains of PANI-Ni were hardly changed compared with PANI. Cyclic voltammogram results indicated that the PANI-Ni film was of excellent electrochemical activity.
\end{abstract}

Keywords: polymer synthesis, polyaniline, ferromagnetic, hysteresis loop, nickel

\section{Introduction}

For many years chemists and physicists have striven to synthesize organic materials with both conducting and ferromagnetic properties due to their potential applications in batteries, electricalmagnetic shields, sensors and microwaveabsorbants [1-3]. Several approaches such as electrochemical and in-situ chemical polymerizations have been reported to prepare conducting polymer with ferromagnetic property [4-7]. Wan and coworkers reported a method for synthesizing a composite of $\mathrm{PANI} / \mathrm{Fe}_{3} \mathrm{O}_{4}$ with both conducting and ferromagnetic functions [8]. Aphesteguy et al. reported preparation and characterization of $\mathrm{Ni}-\mathrm{Gd}$ ferrite polyaniline composite of both conducting and ferromagnetic characterizations [9]. Alam et al. reported the effect of ferrofluid concentration on electrical and magnetic properties of the $\mathrm{Fe}_{3} \mathrm{O}_{4} /$ PANI nanocomposites [10]. As far as the compos- ites were concerned, phase separation was a commonplace due to their poor compatibility between polymer matrix and inorganic particles [11]. Rajca and his colleagues reported a ferromagnetic phase below $10 \mathrm{~K}$ in a polymeric form of a high spin state aromatic molecule [12]. Recently Zaidi et al. reported the polymer of PANi-TCNQ (tetracyanoquinodimethane) produced from polyaniline and tetracyanoquinodimethane at ambient temperature. It is difficult to tell whether PANi-TCNQ is a polymer with both conducting and ferromagnetic properties because no conductivity data are available in the literature [13]. Ichinohe and co-workers reported that ferromagnetic poly $(m$-phenylenediamine) was synthesized by using $\mathrm{H}_{2} \mathrm{O}_{2}$ as an oxidant and horseradish peroxidase (HRP) as a catalyst in the mixture of aqueous buffer solution and 1,4-dioxane or in reversed micellar solution, and the obtained polymer was attracted to a permanent

*Corresponding author, e-mail: jqkan@yzu.edu.cn

(c) BME-PT 
magnet, but no conductivity data were available in the paper, either [14]. Niu and co-workers reported the oxidation of $m$-phenylenediamine by using $\mathrm{H}_{2} \mathrm{O}_{2}$ as an oxidant and horseradish peroxidase as a catalyst in $0.02 \mathrm{~mol} \mathrm{dm}^{-3} \mathrm{~B}-\mathrm{R}$ buffer solution, and 2,7-diaminophenazine was only obtained under the selected reaction condition [15]. We tried to duplicate the former results, however, only 2,7diaminophenazine was still obtained and no precipitates were observed in the mixture of aqueous buffer solution and 1,4-dioxane.

Gosk and his colleagues reported that high-spin $\mathrm{Fe}^{3+}$ complex could be formed with imine and amine nitrogen in polyaniline [16]. Sun and coworkers presently reported that $\mathrm{Co}^{2+}$ ion might bind to more than one nitrogen site in a PANI chain or form inter-chain linkage among several adjacent PANI chains by coordination, and both intra-chain and inter-chain connections might lead to a more coil-like conformational change or a more twisted aggregation of PANI chains [17].

This paper will present a new and easy approach that synthesizes both conducting and ferromagnetic polyaniline with trace metal ions at ambient temperature. This is because there are unpaired electrons and vacant orbit in some metal ions [18] and donating ability of the lone-pair electrons on the nitrogen atom in conducting polyaniline chains [19]. When metal ions are added in the synthesized system of conducting polyaniline, the metal ions may interact with the nitrogen atoms in conducting polyaniline chains and form with both conducting and ferromagnetic polymers. The method is different from what Wan and co-workers described [8] and the resulting product is a complex rather than a composite entrapped with $\mathrm{Fe}_{3} \mathrm{O}_{4}$ as core and polyaniline as shell.

\section{Experimental methods}

\subsection{Materials}

The monomer aniline (reagent grade) was distilled into colorless under reduced pressure before use. Ammonium peroxydisulphate (APS), N,Ndimethylformamide (DMF) and other chemicals were reagent grade and used as received without further treatment. All of the aqueous solutions were prepared with double distilled water.

\subsection{Polymerization}

The PANI were synthesized in $0.1 \mathrm{dm}^{3}$ of solution containing $0.5 \mathrm{~mol} \mathrm{dm}^{-3} \mathrm{HCl}, 0.1 \mathrm{~mol} \mathrm{dm}^{-3}$ aniline, $4 \mathrm{mg}$ HRP, with and without $0.1 \mathrm{~mol} \mathrm{dm}^{-3}$ $\mathrm{NiCl}_{2} \cdot 6 \mathrm{H}_{2} \mathrm{O}$. APS of same molar ratio as aniline was added into the solution. The chemical polymerization of aniline was carried out for 24 hours at $30^{\circ} \mathrm{C}$. The solution was stirred in an ultrasonic bath. The products were firstly separated by filtration, then washed with both double distilled water and ethanol, and finally dried at $65^{\circ} \mathrm{C}$ for 24 hours.

\subsection{Analysis}

The UV-vis spectra of all samples were obtained on UV-2550 spectrometer (Shimadzu) in the range of $250 \sim 900 \mathrm{~nm}$. DMF was used as a solvent. Fourier Transform Infrared (FTIR) spectra were taken on a Tensor 27 FTIR spectrometer (Bruker) using $\mathrm{KBr}$ pellets at a resolution of $4 \mathrm{~cm}^{-1}$. The morphologies of products were observed using a transmission electron microscope (TEM, TENCNAI-12). Conductivity of the resulting products was measured by using standard four-probe method. The standard deviation was less than $2.0 \%$ in our experiments using an YJ8312 model current source (Shanghai Huguang Instrument Works, China) and M92A digital multimeter (Mastech). Wide-angle X-ray diffraction patterns for the powder samples were taken on a M03XHF22 diffractometer (Mac Science, Japan) using $\mathrm{Cu}-\mathrm{K}_{\alpha}$ radiation $(\lambda=1.541 \AA)$, which was used to analyze the crystallinity of polyaniline. The hysteresis loops were obtained on a Vibrating Sample Magnetometer (VSM, USA). The Electron Paramagnetic Resonance (EPR) spectra were carried out by using an A300-10/12 spectrometer (Bruker) operating in $X$-band $(9.856 \mathrm{GHz})$. The microwave power was set at $0.2 \mathrm{~mW}$ and the amplitude was controlled at $2.00 \mathrm{G}$.. X-ray photoelectron spectroscopy (XPS) was performed with a VG EscaLab 220i spectrometer using a standard $\mathrm{Al} K_{\alpha} \mathrm{X}$-ray source $(300 \mathrm{~W})$. The cyclic voltammogram (CV) was performed on a CHI 407 electroanalysis apparatus. An electrolytic cell for cyclic voltammetry consisted of a PANI working electrode, a platinum counter electrode and a saturated calomel electrode (SCE). 


\section{Results and discussion}

\subsection{Electrical and magnetic properties}

Figure 1 shows the plots of magnetization $(\boldsymbol{M})$ against the magnetic field $(\boldsymbol{H})$ of PANI (curve a), deprotonated PANI with $\mathrm{NH}_{3} \cdot \mathrm{H}_{2} \mathrm{O}$ (curve b) and PANI-Ni (curve c), respectively. Curves (a) and (b) indicate that PANI and deprotonated PANI with $\mathrm{NH}_{3} \cdot \mathrm{H}_{2} \mathrm{O}$ are not ferromagnetic. Curve (c) clearly exhibits hysteresis loop at about $300 \mathrm{~K}$, which indicates that the PANI-Ni is ferromagnetic. The inset in Figure 1 is $\boldsymbol{M}-\boldsymbol{H}$ plot of $\mathrm{NiCl}_{2} \cdot 6 \mathrm{H}_{2} \mathrm{O}$ under the same condition. It can be seen from the inset that the magnetization of $\mathrm{NiCl}_{2} \cdot 6 \mathrm{H}_{2} \mathrm{O}$ increases linearly

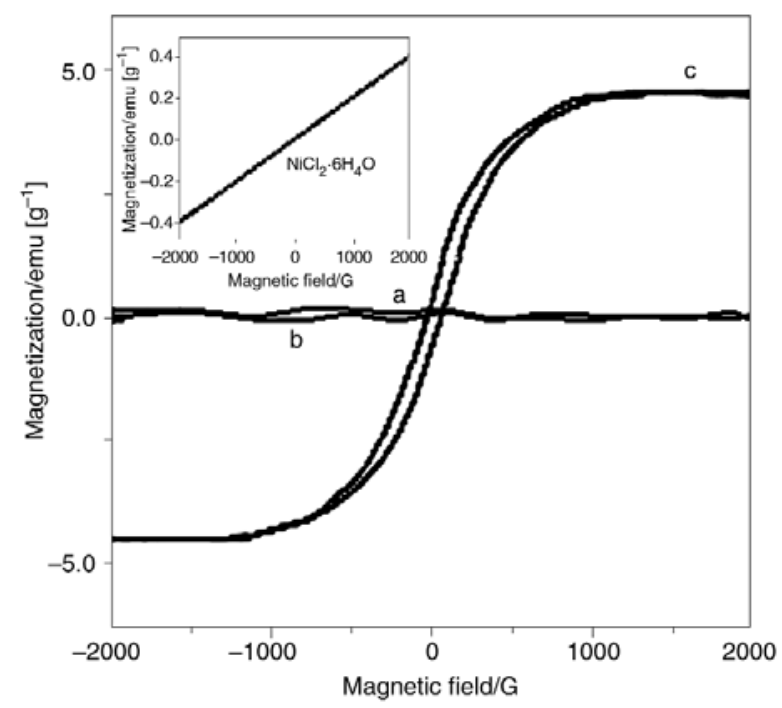

Figure 1. $\boldsymbol{M}$ - $\boldsymbol{H}$ plot of PANI (a), deprotonated PANI (b) and PANI-Ni (c)

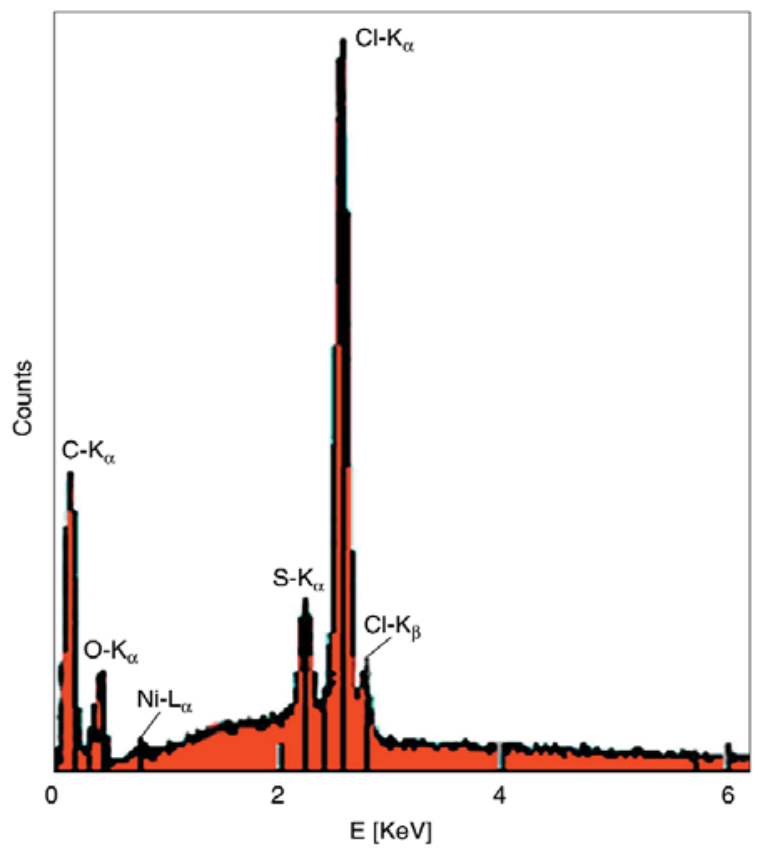

Figure 2. XPS spectrum of PANI-Ni with increasing applied magnetic field and there is no hysteresis loop at ambient temperature, which imply that $\mathrm{NiCl}_{2} \cdot 6 \mathrm{H}_{2} \mathrm{O}$ is just a paramagnetic salt. According to the result of XPS (Figure 2) of PANI$\mathrm{Ni}$, there is only trace amount of nickel in the PANI-Ni $(<0.5 \%)$, so the nickel ions can't give noticeable contribution to the ferromagnetic property of the PANI-Ni. The result of EPR shows that there are unpaired electrons in the PANI-Ni and the spin density of the resulting product is $1.09 \cdot 10^{19}$ spins $^{-1}$. It is well known that the ferromagnetic state not only requires (quasilocal) magnetic moments but also requires that the moments remain mutually aligned [20]. The results of VSM and EPR show that PANI-Ni is in keeping with the requirement of ferromagnetic state. The result of TEM in Figure 3 shows that the morphology of PANI-Ni (Figure $3 \mathrm{~b}$ ) is very similar with that of the PANI-Co, both of which are quasi-spherical morphologies. Sun and co-workers thought that $\mathrm{Co}^{2+}$ ion might bind to more than one nitrogen site in a PANI chain or form inter-chain linkage among several adjacent PANI chains by coordination, and both intra-chain and inter-chain connections might lead to a more coil-like conformational change or a more twisted aggregation of PANI chains [17]. It is deduced that the interaction between $\mathrm{Ni}^{2+}$ and PANI chains may be favorable to form ferromagnetic domain. The forming mechanism of conductive ferromagnetic PANI-Ni is suggested as Figure 4 [21]. However, more researches are needed to understand the behavior better.

It is seen from Figure 1 that the saturation magnetization $\left(\boldsymbol{M}_{s}\right)$ and the coercive force $\left(\boldsymbol{H}_{c}\right)$ of the PANI-Ni are $4.5 \mathrm{emu} \mathrm{g}^{-1}$ (electromagnetic unit per gram) and about $55 \mathrm{Oe}$, respectively. The low coercive value implies that the PANI-Ni is a soft ferromagnetic material [14].

It is difficult to understand that the content of nickel in the resulting PANI is hardly related with the con-

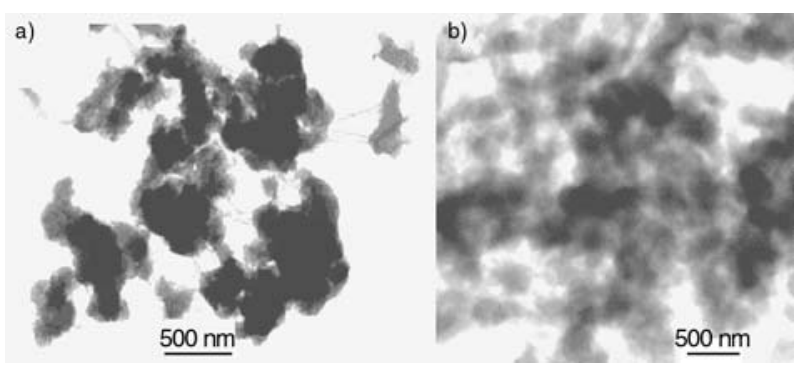

Figure 3. TEM morphology of PANI (a) and PANI-Ni (b) 

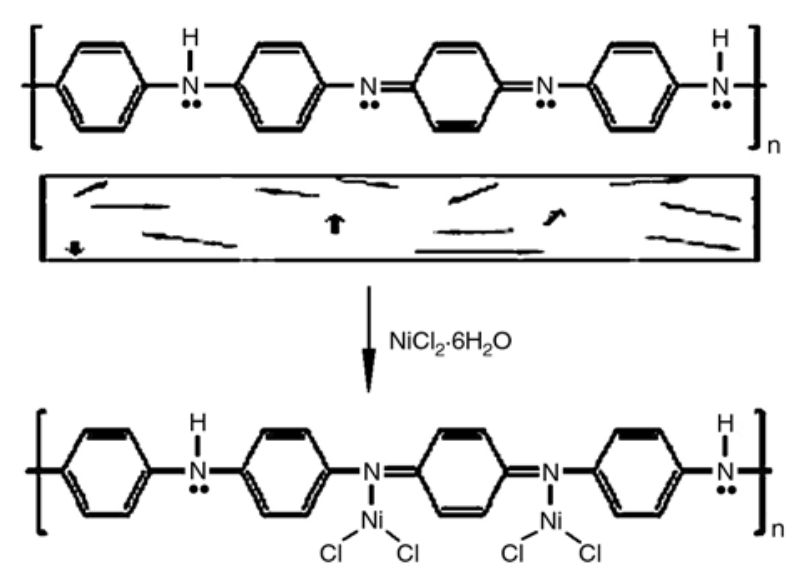

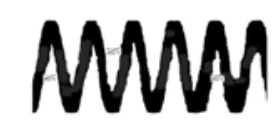

Spiral structure

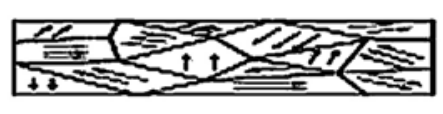

Magnetic domain
Figure 4. The possible forming mechanism of ferromagnetic PANI-Ni

centration of $\mathrm{NiCl}_{2}$ in reaction system. We consider that this phenomenon can be easily explained with Peauson's theory of Hard and Soft acids and bases (HSAB), Hard acids prefer to bind to hard bases and soft acids prefer to bind to soft bases [22]. Based on similar system of HSAB's classification reported in the literature, $\mathrm{H}^{+}$[22] and PANI [23] are hard acid and hard base respectively, so PANI can be easily doped by $\mathrm{H}^{+}$. $\mathrm{Ni}^{2+}$ is borderline acids [22], so PANI is just able to be coordinated with trace $\mathrm{Ni}^{2+}$ compared with $\mathrm{H}^{+}$.

The conductivities of PANI-Ni and PANI are 0.22 and $0.13 \mathrm{~S} \mathrm{~cm}^{-1}$, respectively. It indicates that $\mathrm{Ni}^{2+}$ has little effect on the conductivity of PANI.

As it is mentioned above, PANI-Ni is a conducting and ferromagnetic material at ambient temperature.

\subsection{UV-vis spectra and FTIR spectra}

Figure 5 is the UV-vis spectra of PANI (curve a), PANI-Ni (curve b) dissolved in DMF, and solid PANI (curve c), respectively. It is seen obviously from Figure 5a and Figure 5b that there are two absorption bands in the two curves: one location is $\sim 350 \mathrm{~nm}$ and the other is $\sim 600 \mathrm{~nm}$. It was reported that PANI showed two strong absorptions at 320 340 and 600 660 nm respectively [24]. The first band was assigned as $\pi-\pi^{*}$ transition and the other were associated with the transition of benzenoid rings into quinoid rings. In fact, the PANI we synthesized is in the protonated form, the

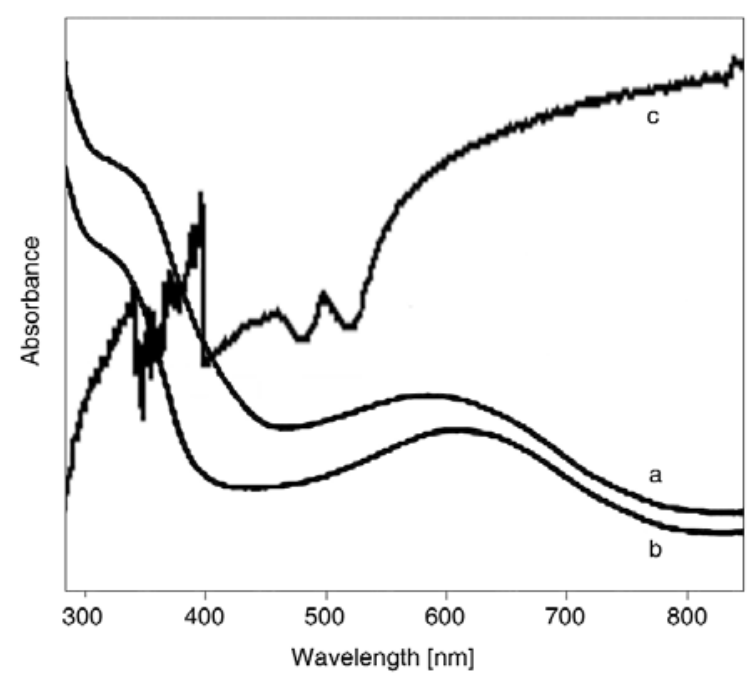

Figure 5. UV-vis spectra of PANI (a), PANI-Ni (b) and solid PANI (c)

absorption band of the transition of benzenoid rings into quinoid rings should be at more than $700 \mathrm{~nm}$. This may be due to the presence of the hydrogenbonding interaction of the $\mathrm{C}=\mathrm{O}$ group in $\mathrm{DMF}$ with the $\mathrm{NH}$ group in PANI. Since the $\mathrm{C}=\mathrm{O}$ group can interact or form hydrogen bond with the dopant, which could prevent the PANI from acid-doping [25]. This can be confirmed by a broad absorption band at more than $700 \mathrm{~nm}$ in the UV-vis spectrum of solid PANI (curve c).

Compared curve (b) with curve (a) in Figure 5, the peak of quinoid ring transition shifts from 585 to $621 \mathrm{~nm}$ when $\mathrm{Ni}^{2+}$ is added in the reactive system. The reason may be that 'compact coil' of PANI-Ni chain make the energy gap of quinoid ring transition narrower, thus transition of electrons becomes easier. The peak of $\pi-\pi^{*}$ transition in PANI seldom shifts, so it can be inferred that the interaction of both $\mathrm{Ni}^{2+}$ and the nitrogen atoms on the quinoid ring is predominant.

Figure 6 shows the FTIR spectra of PANI (curve a) and PANI-Ni (curve b) respectively. IR spectra over $2500 \mathrm{~cm}^{-1}$ are omitted in Figure 6 because they are really identical with and without $\mathrm{NiCl}_{2}$. 1570 and $1490 \mathrm{~cm}^{-1}$ are attributed to the stretching vibrations of $\mathrm{N}=\mathrm{Q}=\mathrm{N}$ ring and $\mathrm{N}-\mathrm{B}-\mathrm{N}$ ring respectively. The peak at $1300 \mathrm{~cm}^{-1}$ corresponds to $\mathrm{C}-\mathrm{N}$ stretching vibration. The peaks at 1109 and $800 \mathrm{~cm}^{-1}$ can be attributed to bands characteristic of B-NH-Q or B-NH-B bonds, and out-of-plane bending vibration of $\mathrm{C}-\mathrm{H}$ of benzene rings (where $\mathrm{B}$ refers to the benzenic-type rings and $\mathrm{Q}$ refers to 


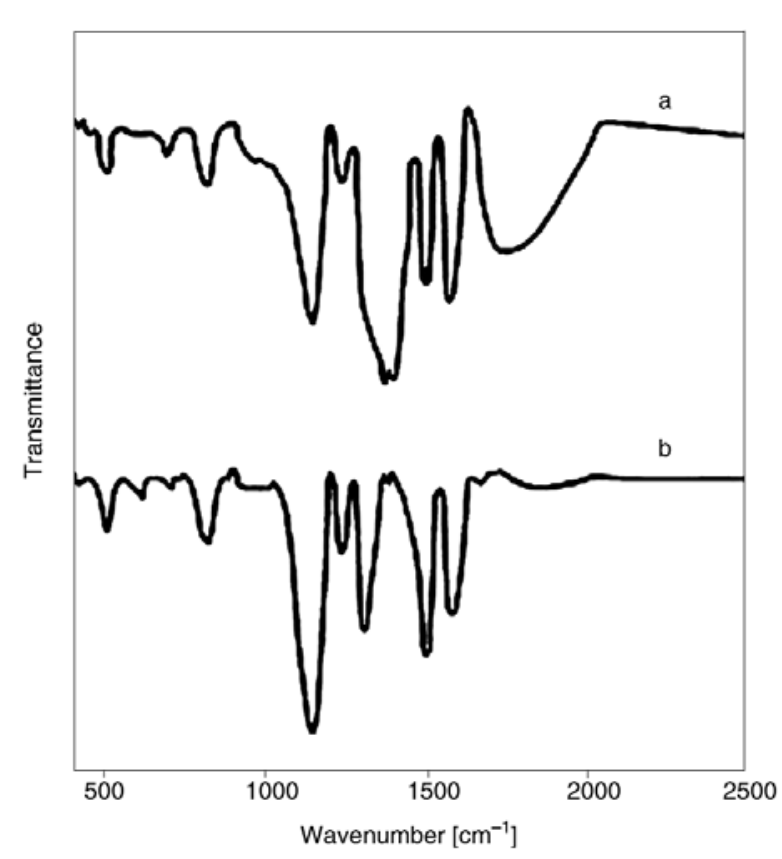

Figure 6. FTIR spectra of PANI-Ni (a) and PANI (b)

the quinonic-type rings) $[24,26,27]$. It is confirmed by our result (as shown in Figure 6) that band at $1400 \mathrm{~cm}^{-1}$ assigned to $\mathrm{N}-\mathrm{H}$ bending mode shifts to $1303 \mathrm{~cm}^{-1}$ when $\mathrm{Ni}^{2+}$ is added in the reactive system of PANI, which indicates that the $\mathrm{Ni}^{2+}$ can interact with nitrogen atoms of the PANI chains.

It can be inferred from the results of UV-vis and FTIR spectra that backbone chain of PANI-Ni hardly changes compared with that of PANI. So the PANI-Ni is a complex polymer under the aforementioned condition.

\subsection{Cyclic voltammogram}

Figure 7 is the cyclic voltammogram of PANI film (curve b) and PANI-Ni film (curve a) in $0.5 \mathrm{~mol} \mathrm{dm}^{-3} \mathrm{HCl}$ aqueous solution in the range from -0.2 to $0.9 \mathrm{~V}$ at $60 \mathrm{mV} \cdot \mathrm{s}^{-1}$. It can be seen obviously from Figure 7 that the PANI-Ni is of electrochemical activity.

There are three pairs of redox peaks in the two curves. Peaks 1 and 3 have been identified as oxidation of PANI to its radical cation (polaron) and further oxidation to quinoid (bipolaron) states, respectively [28]. Peaks $1^{\prime}$ and $3^{\prime}$ correspond to the inverse process of Peaks 1 and 3 [29, 30]. The 'middle' peaks 2 and 2 ' have been attributed to the presence of ortho-coupled polymers [31] and the

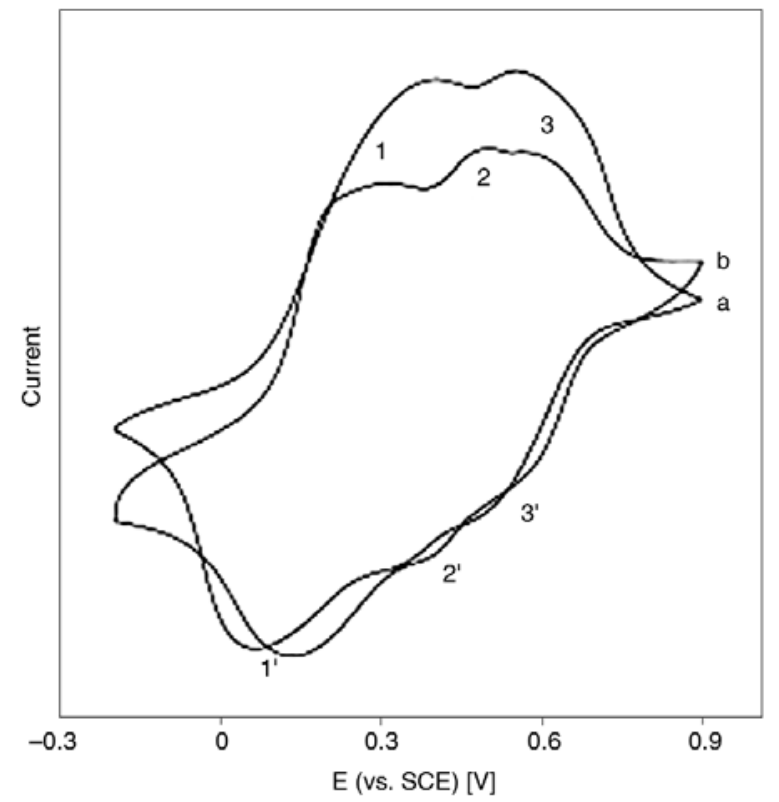

Figure 7. The cyclic voltammograms of PANI (a) and PANI-Ni film (b) $0.5 \mathrm{~mol} \mathrm{dm}^{-3} \mathrm{HCl}, 60 \mathrm{mV} / \mathrm{s}$

degradation of PANI (soluble species such as benzoquinone and hydroquinone) [28].

Compared curve (b) with curve (a), the two curves are similar in shape. The oxidation-reduction peaks show slight shifts. For example, the anodic peaks at 0.05 and $0.33 \mathrm{~V}$ for PANI film shift to 0.13 and $0.40 \mathrm{~V}$ for the PANI-Ni, respectively. These shifts may result from the interaction between $\mathrm{Ni}^{2+}$ and PANI chains.

\subsection{X-ray diffraction spectra}

Figure 8 shows the XRD patterns of $\mathrm{NiCl}_{2} \cdot 6 \mathrm{H}_{2} \mathrm{O}$ (curve c) and PANI (curve b) and PANI-Ni (curve a). The X-ray diffractogram of $\mathrm{NiCl}_{2} \cdot 6 \mathrm{H}_{2} \mathrm{O}$ (curve c) indicates the characteristic diffraction peaks of $\mathrm{NiCl}_{2} \cdot 6 \mathrm{H}_{2} \mathrm{O}$ centered at $2 \theta=16.5,18.6$, $29.2,32.8,35.5,37.5^{\circ}$. However, these diffraction peaks didn't appear in the XRD patterns of PANINi. This may be because there is only trace amount of nickel in PANI-Ni. The PANI (curve a) and PANI-Ni (curve b) have a broad hump peak at $2 \theta=$ $20^{\circ}$, which shows that PANI-Ni is an amorphous polymer [32]. This result is in agreement with those reported in literature [33]. Based on the similar diffractogram of curves (a) and (b) in Figure 8, it maybe because there is hardly effect of $\mathrm{Ni}^{2+}$ on the crystallinity of PANI-Ni. 


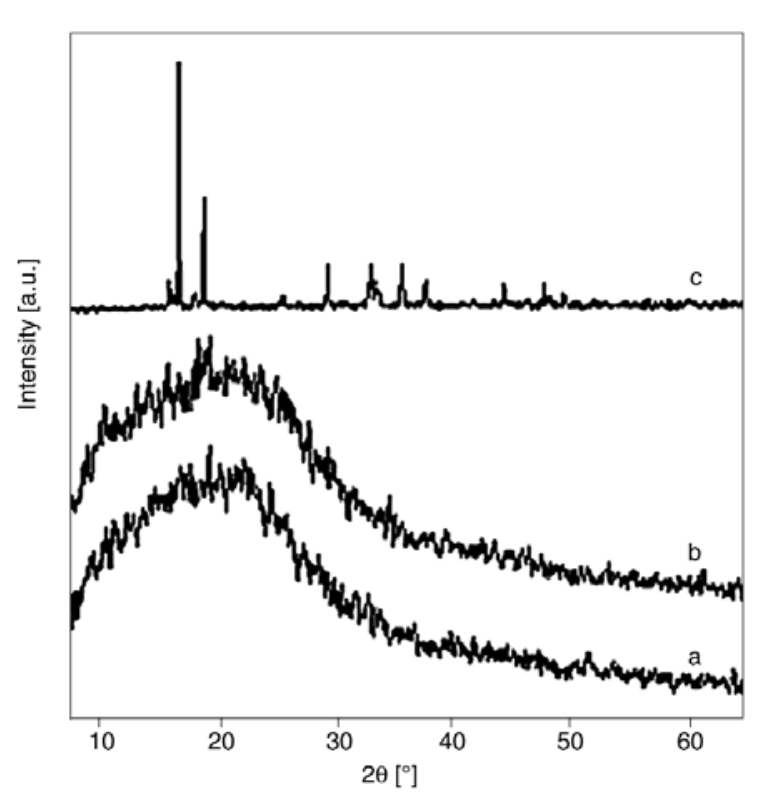

Figure 8. XRD pattern of PANI-Ni (a), PANI (b) and $\mathrm{NiCl}_{2} \cdot 6 \mathrm{H}_{2} \mathrm{O}(\mathrm{c})$

\section{Conclusions}

The conductive and ferromagnetic PANI-Ni is synthesized successfully in the system containing $\mathrm{NiCl}_{2}$ at ambient temperature. The result of VSM indicates that PANI-Ni is of ferromagnetism at about $300 \mathrm{~K}$. The UV-vis and FTIR spectra show that the structure of backbone chains of PANI-Ni hardly changes compared with PANI. The PANI$\mathrm{Ni}$ is a complex polymer rather than a composite.

\section{Acknowledgements}

This project was supported by National Science Foundation of China (No.20673095).

\section{References}

[1] Shen P. K., Huang H. T., Tseug A. C. C.: A study of tungsten trioxide and polyaniline composite films. Journal of The Electrochemical Society, 139, 18401845 (1992). DOI: $10.1149 / 1.2069508$

[2] Kawaguch H.: Functional polymer microspheres. Progress in Polymer Science, 25, 1171-1210 (2000). DOI: 10.1016/S0079-6700(00)00024-1

[3] Kalinina O., Kumacheva E.: A ‘core-shell' approach to producing 3D polymer nanocomposites. Macromolecules, 32, 4122-4129 (1999).

DOI: $\underline{10.1021 / \mathrm{ma} 9815830}$
[4] Bidan G., Jarjayes O., Fruchart J. M., Hannecart E.: New nanocomposites based on 'tailor dressed' magnetic particles in a polypyrrole matrix. Advanced Materials, 61, 52-155 (1994).

DOI: $10.1002 /$ adma.19940060213

[5] Yan F., Xue G., Chen J.: Lu Y.: Preparation of a conducting polymer/ferromagnet composite film by anodic-oxidation method. Synthetic Metals, 123, 1720 (2001).

DOI: $10.1016 / \mathrm{S} 0379-6779(00) 00572-5$

[6] Liu J., Wan M. X.: Composites of polypyrrole with conducting and ferromagnetic behaviors. Journal of Polymer Science Part A: Polymer Chemistry, 38, 2734-2739 (2000).

DOI: $10.1002 / 1099-0518(20000801) 38: 15<2734:$ :

$$
\text { AID-POLA130>3.0.CO;2-R }
$$

[7] Wan M., Li J. C.: Synthesis and electrical-magnetic properties of polyaniline composites. Journal of Polymer Science Part A: Polymer Chemistry, 36, 2799 2805 (2003).

DOI: $10.1002 /($ SICI $) 1099-0518(19981115) 36: 15<$ 2799::AID-POLA17>3.0.CO;2-1

[8] Wan M., Li W.: A composite of polyaniline with both conducting and ferromagnetic functions. Journal of Polymer Science Part A: Polymer Chemistry, 35, 2129-2136 (1997).

DOI: 10.1002/(SICI)1099-0518(199708)35:11<2129:: AID-POLA2>3.0.CO;2-T

[9] Aphesteguy J. C., Bercoff P. G., Jacobo S. E.: Preparation of magnetic and conductive $\mathrm{Ni}-\mathrm{Gd}$ ferritepolyaniline composite. Physica B: Physics of Condensed Matter, 398, 200-203 (2007). DOI: $\underline{10.1016 / j . p h y s b .2007 .04 .018}$

[10] Alam J., Riaz U., Ahmad S.: Effect of ferrofluid concentration on electrical and magnetic properties of the $\mathrm{Fe}_{3} \mathrm{O}_{4} / \mathrm{PANI}$ nanocomposites. Journal of Magnetism and Magnetic Materials, 314, 93-97 (2007). DOI: $10.1016 / \mathrm{j} . j \mathrm{mmm} .2007 .02 .195$

[11] Wang H., Xu P., Meng S., Zhong W., Du W., Du Q.: Poly(methylmethacrylate)/silica/titania ternary nanocomposites with greatly improved thermal and ultraviolet-shielding properties. Polymer Degradation and Stability, 91, 1455-1461 (2006). DOI: 10.1016/j.polymdegradstab.2005.10.008

[12] Rajca A., Wongsriratanakul J., Rajca S.: Magnetic ordering in an organic polymer. Science, 294, 1503 1505 (2001). DOI: $\underline{10.1126 / \text { science. } 1065477}$

[13] Zaidi N. A., Giblin S. R., Terry I. Monkman A. P.: Room temperature magnetic order in an organic magnet derived from polyaniline. Polymer, 45, 5683-5689 (2004). DOI: 10.1016/j.polymer.2004.06.002

[14] Ichinohe D., Muranaka T., Kise H.: Oxidative polymerization of phenylenediamines by enzyme and magnetic properties of the products. Journal of Applied Polymer Science, 70, 717-721 (1998). DOI: 10.1002/(SICI) 1097-4628(19981024)70:4<717:: AID-APP11>3.0.CO;2-U 
[15] Niu S. Y., Jiao K.: Studies on the oxidation of m-phenlene diamine by $\mathrm{H}_{2} \mathrm{O}_{2}$ catalyzed by horseradish peroxidase. Acta Chimica Sinica, 58, 617-621 (2000).

[16] Gosk J. B., Kulszewicz-Bajer I., Twardowski A.: Magnetic properties of polyaniline doped with $\mathrm{FeCl}_{3}$. Synthetic Metals, 156, 773-778 (2006). DOI: 10.1016/j.synthmet.2006.02.003

[17] Tao S., Hong B., Kerong Z.: An infrared and Raman spectroscopic study of polyanilines co-doped with metal ions and $\mathrm{H}^{+}$. Spectrochimica Acta Part A: Molecular and Biomolecular Spectroscopy, 66, 1364-1368 (2007). DOI: $10.1016 /$ j.saa.2006.08.011

[18] Xiao H-P., Morsali A.: A new three-dimensional polymeric $\mathrm{Pb}^{\mathrm{II}}$ complex involving holo- and hemidirected coordination spheres. Solid State Sciences, 9, 155158 (2007).

DOI: 10.1016/j.solidstatesciences.2006.09.005

[19] Trivedi D. C.: Observation of ferromagnetism in polyaniline. Synthetic Metals, 121, 1780-1781 (2001).

DOI: 10.1016/S0379-6779(00)00759-1

[20] Billas I. M. L., Chatelain A., de Heer W. A.: Magnetism from the atom to the bulk in iron, cobalt, and nickel clusters. Science, 265, 1682-1684 (1994). DOI: $10.1126 /$ science.265.5179.1682

[21] Zhang Y., Zhu C., Kan J.: Synthesis and characterization of ferromagnetic polyaniline with conductivity in an applied magnetic field. Journal of Applied Polymer Science, 109, 3024-3029 (2008). DOI: $10.1002 / a p p .28414$

[22] Pearson R. G.: Hard and soft acids and bases, Part I: Fundamental principles. Journal of Chemical Education, 45, 581-587 (1968).

[23] Chehimi M. M., Abel M-L., Perruchot C., Delamar M., Lascelles S. F., Armes S. P.: The determination of the surface energy of conducting polymers by inverse gas chromatography at infinite dilution. Synthetic Metals, 104, 51-59 (1999). DOI: $10.1016 / \mathrm{S} 0379-6779(99) 00040-5$

[24] Laska J., Widlarz J.: Spectroscopic and structural characterization of low molecular weight fractions of polyaniline. Polymer, 46, 1485-1495 (2005). DOI: $10.1016 /$ j.polymer.2004.12.008
[25] Chen S. A., Lee H. T.: Polyaniline plasticized with 1methyl-2-pyrrolidone: Structure and doping behavior. Macromolecules, 26, 3254-3261(1993).

DOI: $10.1021 / \mathrm{ma} 00065 \mathrm{a} 002$

[26] Zeng X-R., Ko T-M.: Structure - conductivity relationships of iodine-doped polyaniline. Journal of Polymer Science: Part B: Polymer Physics, 35, 1993 2001 (1997).

DOI: $10.1002 /($ SICI) 1099-0488(19970930)35:13< 1993::AID-POLB1>3.0.CO;2-O

[27] Cheng D. M., Ng S. C., Chan H. S. O.: Morphology of polyaniline nanoparticles synthesized in triblock copolymers micelles. Thin Solid Films, 477, 1923(2005).

DOI: $\underline{10.1016 / j . t s f .2004 .08 .105}$

[28] Shim Y-B., Won M-S., Park S-M.: Electrochemistry of conductive polymers VIII: In situ spectroelectrochemical studies of polyoniline growth mechanisms. Journal of The Electrochemical Society, 137, 538-544 (1990).

DOI: $\underline{10.1149 / 1.2086494}$

[29] Stilwell D. E., Park S. M.: Electrochemistry of conductive polymers II. Electrochemical studies on growth properties of polyaniline. Journal of the Electrochemical Society, 135, 2254-2262 (1988). DOI: $10.1149 / 1.2096248$

[30] Yan B., Yang J., Li Y., Cao Y.: Electrochemical adsorption of hydrogen and various ions on polyaniline film. Reactions concerning the first pair of cyclic voltammetric peaks. Synthetic Metals, 44, 189-197 (1991). DOI: $10.1016 / 0379-6779(91) 91834-W$

[31] Hand R. L., Nelson R. F.: Anodic oxidation pathways of N-alkylanilines. Journal of the American Chemical society, 96, 850-860 (1974). DOI: $10.1021 / \mathrm{ja} 00810 \mathrm{a} 034$

[32] Yavuz O., Ram M. K., Aldissi M., Poddar P., Hariharan S.: Synthesis and the physical properties of MnZn ferrite and NiMnZn ferrite-polyaniline nanocomposite particles. Journal of Materials Chemistry, 15, 810-817 (2005). DOI: $10.1039 / \mathrm{b} 408165 \mathrm{j}$

[33] Pouget J. P., Jozefowicz M. E., Epstein A. J., Tang X., MacDiarmid A. G.: X-ray structure of polyaniline. Macromolecules, 41, 723-789 (1991). DOI: $10.1021 / \mathrm{ma} 00003 \mathrm{a} 022$ 